\title{
Histopathological Image Analysis: Path to Acceptance through Evaluation
}

\author{
Metin N. Gurcan ${ }^{1}$
}

1. Department of Biomedical Informatics, OSU, Columbus, $\mathrm{OH}$

It is widely believed that digital imaging will become the standard practice in pathology. When digital pathology is adopted in clinical practice, it will bring in several advantages including access to some innovative technologies such as computer-aided diagnosis (CAD). CAD will enable pathologists to perform their tasks more efficiently, consistently and accurately.

We have witnessed a great progress in CAD development for pathology in the last decade. Our group as well as several groups in the world first tackled the problems associated with computational and human factor aspects of digital pathology [1-6] as pathological images tend to be relatively large and pathologists are used to reviewing their images under the microscope. The large size of the images and their complexity often require processing them in a novel multi-resolution analysis framework, which mimics the pathologists' review of these types of cases [7-9]. In these approaches, we have developed algorithms for the automated selection of the appropriate resolution [8-10], and for processing multiresolution images [11]. The implementation may involve a single classifier, or a combination of classifiers to better model the complex domain knowledge [9].

Microscopic images contain complex structures and are subject to inter- and intra-institutional variation due to preparation (i.e. differences in fixation type and length, tissue processing protocols, histologic section thickness, and staining). We have developed several segmentation algorithms to adapt to the complexity of these problems, e.g. Expectation-Maximization based segmentation [12]. We have also developed and adapted several transform based features for the analysis of histopathological images. Most of the currently used classification systems use the inherent color and texture information in the images to identify areas of disease to determine its extent and quality [13].

Once a CAD system is developed, it needs to be carefully evaluated prior to its use in clinics. Evaluation of developed CAD systems is critical to their acceptance by not only regulatory bodies but also by pathologists who will use such systems. Several CAD researchers have adopted an evaluation strategy that we call an "open loop" evaluation (OLE). In this evaluation technique, pathologists annotate a set of images, which are then divided into independent training and test sets. The computer algorithm is developed using the training set and tested on an independent set (i.e. test set). We have been evaluating our image analysis algorithms in a "closed loop" environment (Figure 1). In this environment, similar to OLE, the computer algorithm is trained on a set of annotated cases (i.e. training cases). Then, it is evaluated on an independent test set by pathologists who will have a chance to consult with the computer outcome; they are free to change their original decision or stick to it afterwards. [14] 


\section{References:}

[1] B. Cambazoglu et al, "Efficient processing of pathological images using the grid: Computer-aided prognosis of neuroblastoma," in Proceedings of Fifth International Workshop on Challenges of Large Applications in Distributed Environments (CLADE), Monterey Bay, CA, 2007, pp. 35-41.

[2] A. Ruiz et al, "Pathological image analysis using the GPU: Stroma classification for neuroblastoma," 2007 IEEE International Conference on Bioinformatics and Biomedicine, Proceedings, pp. 78-85, 2007. [3] J. Saltz et al, Computer, vol. 41, pp. 58-66, Nov 2008.

[4] G. Teodoro et al, "Coordinating the Use of GPU and CPU for Improving Performance of Compute Intensive Applications," 2009 IEEE International Conference on Cluster Computing and Workshops, pp. 437-446, 2009.

[5] S. Ali and A. Madabhushi, Journal of pathology informatics, vol. 2, p. 13, 2011.

[6] X. Qi et al, "Robust Segmentation of Overlapping Cells in Histopathology Specimens Using Parallel Seed Detection and Repulsive Level Set," Biomedical Engineering, IEEE Transactions on, pp. 1-1, 2011.

[7] J. Kong et al, 2007 IEEE International Conference on Image Processing, Vols 1-7, pp. 2777-2780, 2007.

[8] J. Kong et al, SPIE Medical Imaging 2008: Computer-Aided Diagnosis, vol. 6915, pp. pp. 452-460, 2008.

[9] J. Kong et al, Pattern Recognition, vol. 42, pp. 1080-1092, 2009.

[10] O. Sertel et al, Pattern Recognition, vol. 42, pp. 1093-1103, Jun 2009.

[11] O. Sertel et al, SPIE Medical Imaging 2008: Computer-Aided Diagnosis, vol. 6915, pp. 44-55, 2008.

[12] J. Kong et al, 2007 4th IEEE International Symposium on Biomedical Imaging : Macro to Nano, Vols 1-3, pp. 61-64, 2007.

[13] M. Gurcan et al, IEEE Reviews in Biomedical Engineering, vol. 2, pp. 147-171, 2009.

[14] The project described was supported in part by Awards Number U24CA199374 (PIs: Gurcan, Madabushi, Martel), and U01 CA198945 (PI: Bilgin) from the National Cancer Institute and by Number R56 AI111823 (PIs: Campos-Neto, Beamer) from the National Institute of Allergy and Infectious Diseases. The content is solely the responsibility of the authors and does not necessarily represent the official views of the National Cancer Institute, the National Institute of Allergy and Infectious Diseases, or the National Institutes of Health.

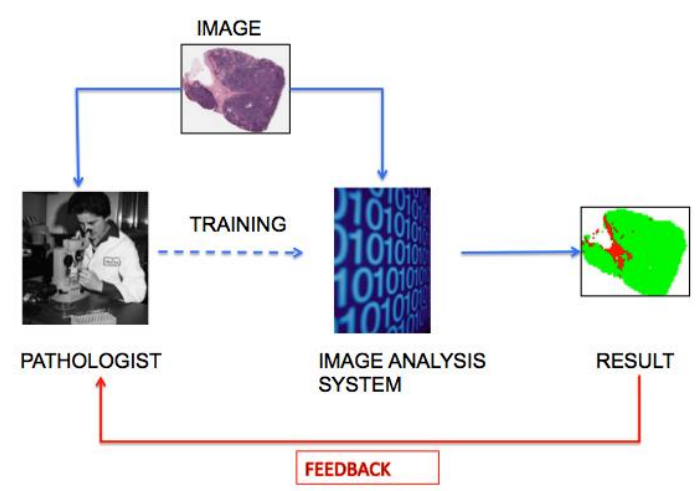

Figure 1. Closed loop evaluation of a CAD system. 